\title{
J ust-in-time training for nurse administration of anesthetic cream application
}

\author{
Deborah R. Liu ${ }^{1}$, Armstrong Hao ${ }^{2}$, Vincent J. Wang ${ }^{1}$ \\ 1. Division of Emergency Medicine, Children's Hospital Los Angeles, USC Keck School of Medicine, Los Angeles, United \\ States. 2. Emergency Department, Children's Hospital Los Angeles, Los Angeles, California, United States.
}

Correspondence: Deborah R. Liu. Address: Division of Emergency Medicine, Children's Hospital Los Angeles, USC Keck School of Medicine, 4650 Sunset Blvd, Mailstop \#113, Los Angeles, California, United States. Email: deliu@chla.usc.edu

Received: May 15, 2013

Accepted: July 14, 2013

Online Published: September 3, 2013

DOI : 10.5430/jnep.v4n1p143

URL: http://dx.doi.org/10.5430/jnep.v4n1p143

\begin{abstract}
Introduction: Just-In-Time Training (JITT) is an emerging concept in medical education. Providing a brief educational intervention in the form of a video immediately prior to the patient intervention may be an effective method to teach health care providers. In this pilot study, we sought to determine if pediatric emergency department nurses could effectively apply a topical anesthetic to the lower back for lumbar puncture after watching a JITT video.

Methods: Between October 19, 2011 and November 1, 2011, thirty nurses were asked to complete a questionnaire assessing their comfort level on applying a topical anesthetic prior to lumbar puncture. Accuracy of sham cream placement onto the back of an infant mannequin was assessed pre- and post-JITT video. Self-reported comfort levels in cream placement, cream coverage, and overall accuracy of cream placement were compared both pre- and post-JITT intervention.

Results: There was a statistically significant change in the self-reported comfort level of the subjects in applying the cream post-JITT $(p<.01)$. In addition, there was a statistically significant difference in cream coverage after the JITT $(p<.01)$. Furthermore, subjects who were inaccurate with cream placement pre-JITT were more likely to be accurate post-JITT $(p<.01)$.

Conclusions: JITT is an effective tool in medical education for teaching topical anesthetic cream application onto the back by pediatric emergency department nurses. JITT may offer other possibilities for enhancing medical education.
\end{abstract}

\section{Key words}

Just-in-time training, Medical education, Medical technology

\section{I ntroduction}

Though Just-In-Time Training (JITT) originated in the automotive manufacturing industry, the fundamental concept of JITT can easily be applied to medical education. In a production line, specific components need to be readily available in large enough quantities, without accumulating a surplus of inventory. Managing a surplus is undesirable as it requires extra costs, and if the parts are not used quickly, they may become obsolete. In medical education, the challenge is how to manage the inventory of information we require to provide high quality health care ${ }^{[1]}$. Some information, such as basic knowledge or frequently used concepts, is easily retrieved from our memory. However, newer medical concepts or information we do not use routinely may not be quite as readily recalled. Such information may not be easily accessible 
using the traditional teaching methods of didactic lectures or reading from medical textbooks. Health care providers often require such information in real time, while providing patient care.

JITT has emerged as a relatively new concept in medical education. For the busy clinician, or one practicing in a resource-scarce environment, JITT can provide efficient and focused medical education at the time of patient contact. One can envision the utility of such efficient and effective education in a developing country, where medical personnel and resources may be limited ${ }^{[2,3]}$. Another potential JITT use is for simple procedures which the health care provider may not perform routinely, or for which the provider requires additional focused training. However, there is limited published data to support this.

In this novel pilot study, we sought to demonstrate the potential of JITT for anesthetic application for lumbar puncture. Pediatric emergency department (PED) registered nurses watched a JITT video demonstrating the technique for applying topical anesthetic cream prior to lumbar puncture. In our PED, most nurses were unfamiliar with the steps required to properly apply a topical anesthetic cream to the lower back for lumbar puncture. Those that had applied the medication in the past felt they were not asked to apply the cream frequently enough to become proficient. Our objective was to determine if PED nurses would effectively and accurately apply a topical anesthetic cream to the lower back for lumbar puncture after watching a JITT instructional video.

\section{Methods}

Our PED is located within an urban, tertiary care, free-standing children's hospital and has approximately 70,000 visits per year. Registered nurses in our PED were enrolled as study subjects. The only inclusion criterion was being a registered nurse (RN) in our PED and there were no exclusion criteria. The hospital Committee on Clinical Investigations approved the study.

The study was conducted in a secluded room within the PED, away from all patient care. After informed consent was obtained, the RN completed a brief questionnaire. Data collected included number of years as an RN (grouped as less than one year, 1-3 years, 3-10 years, and greater than 10 years), comfort level in placing anesthetic cream onto a patient's back for lumbar puncture, number of times the RN had previously applied an anesthetic cream to a patient's back for lumbar puncture, and if the RN had ever received any formal (lecture format) or informal (while providing patient care) instruction on how to apply such an anesthetic cream. Subsequently, the RN was asked to apply a sham cream to an infant mannequin's back in preparation for lumbar puncture. The sham cream was a white, odorless lotion dispensed from a $3 \mathrm{ml}$ syringe. The RN was also given an occlusive dressing, thus simulating the topical anesthetic and occlusive dressing found in various topical anesthetic cream preparations.

The RN then watched a 70-second, detailed video illustrating how to apply topical anesthetic cream to an infant mannequin's back for lumbar puncture. The video was created for the study and was uploaded to the publicly available site YouTube $^{\mathrm{TM}}$, and was easily viewed on a computer or smart phone. The JITT video described and demonstrated placing cream over the lower back in a horizontal fashion at the level of the iliac crest, including one vertebral space above and one space below the iliac crest. The video was designed to be as brief as possible while still illustrating the key elements described above. The video was reviewed for accuracy and clarity by two pediatric emergency medicine physicians, one pediatric emergency department nurse, and one other physician (a urologist) who had never placed anesthetic cream onto a patient's back for lumbar puncture in clinical practice.

After viewing the video for the study, the RN was given the opportunity to watch the video as many times as desired. Following the video, the RN was then asked to apply the sham anesthetic cream onto the mannequin's back once again and to rate his/her comfort level in applying the cream.

Immediately following each application of the cream (both pre-JITT and post-JITT), the study investigators assessed the RN's ability to place the cream in the correct position and timed how long it took the RN to apply the cream. To 
standardize the evaluation of cream placement, a clear template overlay was created which outlined the area which should be covered by cream. This template was placed over the application site to assess the overlap between actual and desired cream placement. The desired area was broken into thirds in order to assess the accuracy of cream placement. Thus, the accuracy of cream placement was evaluated as less than one third covered by cream, between one third to two thirds covered, more than two thirds covered but less than $100 \%$ covered, or $100 \%$ covered. In addition, overall accuracy of cream placement for each subject pre- and post-JITT was categorized as a dichotomous variable, accurate versus inaccurate. "Accurate" placement was defined as covering $100 \%$ of the template whereas "inaccurate" was anything less than $100 \%$ covered.

\section{Statistical analysis}

To detect a moderate effect size, with $80 \%$ power and $\alpha=.05$, sample size calculations for a one-tailed test required 28 subjects. The Wilcoxon signed-ranks test was used for non-parametric testing of pre and post-JITT comfort level with applying the cream and for time taken to place the cream pre and post-JITT. The McNemar test, which analyzes a repeated measures design in which each subject gives a binary response twice, once before and once after an intervention occurs, was used to assess if the JITT made a difference in accuracy of cream placement.

\section{Results}

Thirty subjects were enrolled. The number of years of experience as an $\mathrm{RN}$ ranged from less than one year to more than 10 years. Based on the a priori categorizations, the mode (14 subjects) was 3-10 years of experience. Sixteen RNs (53\%) had no previous experience applying an anesthetic cream to a patient's lower back prior to lumbar puncture. Twenty-two RNs (73\%) had never received any instruction on how to apply cream in this scenario. Of the 8 RNs (27\%) who had received education, 2 obtained the information by formal lectures while 6 received instruction in an informal setting.

Prior to the JITT intervention, 8 RNs (27\%) felt that they were "not comfortable" with applying the cream, 12 (40\%) were "somewhat comfortable", 7 (23\%) were "very comfortable", and 3 (10\%) were "extremely comfortable". Post-JITT, no RNs were "not comfortable", with 5 (17\%) being "somewhat comfortable", 16 (53\%) being "very comfortable", and 9 $(30 \%)$ being "extremely comfortable". The Wilcoxon signed-ranks test indicated that there was a statistically significant change $(p<.01)$ in comfort level after the JITT intervention.

Of the 30 subjects, 28 (93\%) watched the JITT video once and $2(7 \%)$ watched the video twice. All 30 subjects felt that it would be helpful to have more JITT videos for simple nursing procedures and tasks. The Wilcoxon signed-ranks test showed that there was no statistically significant change $(p=.48)$ in time used to place the cream (pre-JITT mean $=46$ seconds, median $=41$ seconds; post-JITT mean $=45$ seconds, median $=42$ seconds).

When assessing the accuracy of cream placement by using the template overlay, two study subjects were excluded from analysis because they did not have their template measurement recorded, but both were observed applying the cream in the correct position both before and after JITT. Thus, out of 28 subjects, the pre-JITT assessment showed 2 RNs (7\%) had less than one third of the proper area covered, $5 \mathrm{RNs}(18 \%)$ covered one third to two thirds of the area, $14 \mathrm{RNs}(50 \%)$ covered more than two thirds but less than $100 \%$ of the area, and 7 RNs (25\%) covered the entire area. Post-JITT video, one RN (4\%) had less than one third of the proper area covered, one RN (4\%) covered one third to two thirds of the area of the area, 3 RNs (11\%) covered more than two thirds but less than $100 \%$ of the area, and 23 RNs ( $82 \%)$ covered the entire area. Using the Wilcoxon signed-ranks test, there was a statistically significant change $(p<.01)$ in cream coverage after the JITT intervention.

Overall cream placement accuracy was also assessed (accurate vs. inaccurate). Of the 28 subjects, 4 (14\%) were inaccurate both before and after the JITT intervention, $16(57 \%)$ were inaccurate before JITT but achieved accuracy after the intervention, $1(4 \%)$ was accurate before JITT but was inaccurate after the intervention, and $9(32 \%)$ achieved accuracy both before and after JITT. The McNemar test was statistically significant $(p<.01)$, which indicated that subjects who were inaccurate before JITT were more likely to achieve accuracy after JITT. 


\section{Discussion}

Just-In-Time Training is a relatively new and emerging concept in medical education and technology. It has the potential to aid the clinician who needs skill acquisition immediately prior to performing a simple procedure, where utilizing conventional methods of didactic lectures or medical textbooks may not be of practical use ${ }^{[4,5]}$. Using JITT may allow the clinician to quickly retrieve such information and then immediately apply this towards patient care at the time of patient contact. This type of education may be particularly effective in the resource-scarce environment of a developing country where clinicians are called upon to perform tasks outside their comfort zone ${ }^{[6,7]}$. While JITT is not meant to replace conventional methods of teaching, it may complement traditional medical education in the clinical setting. In our study, we demonstrated that JITT may be effectively utilized to instruct registered nurses to apply topical anesthetic cream to the back of infants requiring lumbar puncture.

A few published studies have begun to investigate the utility of JITT in medical education. McIntosh et al. used JITT to teach dental splinting techniques to emergency physicians ${ }^{[8]}$. Davis et al showed that a 3-minute instructional video resulted in improvement of chest tube placement skills on a mannequin ${ }^{[9]}$. Most recently, Nishisaki et al. used a JITT intervention for pediatric residents in orotracheal intubation in the pediatric intensive care unit ${ }^{[10]}$.

Our study was a novel application of JITT, assessing the effectiveness of JITT for nurse administration of sham anesthetic cream application prior to lumbar puncture. This task is a multi-step procedure infrequently performed by nurses in our PED, which rendered it ideal for JITT. We showed that the 70-second JITT intervention resulted in a significant change in self-rated comfort level for cream application as well as a significant change in how accurately the cream was applied. There was also a statistically significant change in improved cream coverage post-JITT. In addition, the subjects who were inaccurate in cream placement pre-JITT were more likely to achieve accuracy post-JITT intervention. For clinicians with or without experience in the application of anesthetic cream prior to lumbar puncture, utilizing a brief JITT video has the potential to effectively teach this task.

Our study has several limitations. Because the nurses were asked to self-report their comfort level on cream placement preand post-JITT intervention, this data is inherently subject to reporting bias. Also, while we attempted to objectify the evaluation of accuracy of cream placement by using a template overlay, there remains the potential for human error in this assessment. Our model served as a proxy for actual anesthetic cream placement on infants, and the ultimate test of effectiveness would have been measuring pain scales in clinical practice of lumbar puncture. However, as a novel application of JITT, measuring actual effect in live patients was estimated to be unjustified until demonstrated in other studies. Lastly, our study population consisted of a small subgroup of nurses (i.e., pediatric emergency department nurses). While this may limit the generalizability of our study to all registered nurses, this is unlikely to be meaningful.

\section{Conclusion}

Watching a brief JITT instructional video resulted in pediatric emergency department nurses being able to more effectively place an anesthetic cream onto a mannequin's back prior to lumbar puncture. From a clinical perspective, there may be other multistep procedures performed by physicians, nurses, and technicians in which the execution of such procedures may be improved by JITT intervention. Future study should be directed to improving clinical education through JITT.

\section{References}

[1] Chueh H, Barnett GO. “Just-in-time” Clinical Information. Academic Medicine. 1997; 72: 512-17. http://dx.doi.org/10.1097/00001888-199706000-00016

[2] Chandrasekhar CP, Ghosh J. Information and communication technologies and health in low income countries: the potential and the constraints. Bulletin of the World Health Organization. 2001; 79: 850-855. PMid:11584733 
[3] Siddiqi K, Newell J, Robinson M. Getting evidence into practice: what works in developing countries? International Journal for Quality in Health Care. 2005; 17: 447-453. PMid:15872024 http://dx.doi.org/10.1093/intqhc/mzi051

[4] Williamson JW, German PS, Weiss R, Skinner EA, Bowes F. Health science information management and continuing education of physicians: A survey of U.S. primary care practitioners and their opinion leaders. Ann Intern Med. 1989; 110: 151-60.

PMid:2909205 http://dx.doi.org/10.7326/0003-4819-110-2-151

[5] Gorman PN, Helfand M. Information seeking in primary care: how physicians choose which clinical questions to pursue and which to leave unanswered. Med Decis Making. 1995; 15: 113-119. http://dx.doi.org/10.1177/0272989X9501500203

[6] Plat G, Arditi D. The JIT materials management system in developing countries. Construction Management \& Economics. 2005; 23: 697-712. http://dx.doi.org/10.1080/01446190500041388

[7] Msimangira KAB. Using "Just-In-Time" in developing countries: a case study in Tanzania. Journal of Supply Chain Management. 1993; 29: 43-49.

[8] McIntosh MS, Konzelmann J, Smith J, Kalynych CJ, Wears RL, Schneider H, et al. Stabilization and treatment of dental avulsions and fractures by emergency physicians using just-in-time training. Ann Emerg Med. 2009; 54: 585-592. PMid:19700221 http://dx.doi.org/10.1016/j.annemergmed.2009.06.510

[9] Davis JS, Garcia GD, Wyckoff MM, Alsafran S, Graygo JM, Withum KF, et al. Use of mobile learning module improves skills in chest tube insertion. Journal of Surgical Research. 2012; 177: 21-26. PMid:22487392 http://dx.doi.org/10.1016/j.jss.2012.03.022

[10] Nishisaki A, Donoghue AJ, Colborn S, Watson C, Meyer A, Brown CA, et al. Effect of just-in-time simulation training on tracheal intubation procedure safety in the pediatric intensive care unit. Anesthesiology. 2010; 113: 214-223. PMid:20526179

http://dx.doi.org/10.1097/ALN.0b013e3181e19bf2 